\title{
Défigement des phrasèmes dans la poésie moderne : Étude comparative français-catalan.
}

\author{
Yakubovich, Yauheniya \& Català Guitart, Dolors \\ Universitat Autònoma de Barcelona, laboratoire $f$ LexSem \\ \{Yauheniya.Yakubovich et Dolors.Catala\}@uab.cat
}

\section{Introduction}

Le défigement comme recours stylistique a été utilisé depuis l'époque de la «défense et illustration » des langues nationales, par de grands auteurs comme Rabelais ou Quevedo, par exemple ; c'est, pourtant, l'époque contemporaine qui semble fournir le plus grand nombre d'exemples de manipulations linguistiques servant de principe à des courants littéraires importants (dadaïsme, surréalisme, oulipisme, post-modernisme...). De nos jours, la haute clichéisation de la langue impose aux poètes la révision des moyens expressifs qui ne résident plus dans des combinaisons lexicales régies par la norme. De ce point de vue, le défigement est une sorte de fuite des «griffes » de la normativité langagière, la rupture des paradigmes triviaux, et la quête de nouvelles combinatoires, comme nous le prouvent ces lignes de Desnos :

\author{
Il n'avait pas sa langue dans sa poche \\ Ni la main dans la poche du voisin \\ (Langage cuit, «C'était un bon copain »; Desnos, 1953)
}

Ce défi à la norme linguistique brise le figement mais aussi le met en relief, c'est-à dire, fait évoquer son étymologie, actualise le sens primaire ou ranime la métaphore qui était à l'origine du figement. Cette opération de déchiffrement du défigement, purement métalinguistique, fait appel à la conscience à la fois linguistique et culturelle que les locuteurs d'une langue partagent. Dans le langage littéraire ou celui de la presse et de la publicité, le défigement est chargé de fonctions spécifiques, poétique et conative respectivement, si l'on utilise la terminologie de Jakobson. Dans la poésie, qui est le domaine légitime des " mots en liberté ", le défigement sert à rafraîchir une image poétique, à faciliter la mémorisation et, bien entendu, à tromper l'attente du lecteur. Tous ces effets, ludiques et/ou esthétiques, sont assurés par les mécanismes, parfois assez complexes, de la rupture de la contrainte.

C'est précisément pour l'utilité métalinguistique et les effets stylistiques que le défigement produit dans les textes poétiques, que ses mécanismes doivent, à notre avis, être pris en considération par les linguistes. Dans cet article, donc, nous abordons le défigement en tant que procédé linguistique et recours poétique.

Cette étude fait partie d'une recherche plus vaste dont l'objet est constitué par les phrasèmes défigés dans les textes poétiques contemporains en six langues (français, catalan et espagnol comme composante romane; bélarusse, russe et polonais comme composante slave), c'est-à-dire, dans une perspective contrastive plurilingue. Nos objectifs visent à : 1) analyser un vaste corpus de textes poétiques au moyen des outils conceptuels développés, principalement, dans le cadre de la Théorie Sens-Texte (= TST) ; 2) identifier et systématiser les exemples de défigement dans chacune des six langues; 3) fournir des éléments linguistiques formalisés pour définir les effets stylistiques du défigement dans la poésie moderne.

Nous adoptons ici la même approche mais nous limitons notre analyse à deux langues romanes ${ }^{1}$, le français et le catalan. Pour ce faire, nous structurons notre article de la manière suivante : nous entamons l'étude par un bref état de la question et par l'introduction de l'appareil conceptuel de base ; puis, nous passons aux typologies de défigement que nous avons établies en nous appuyant sur des corpora multilingues de phrasèmes défigés que nous avons constitués, et nous terminons par la description et la comparaison de plusieurs exemples de phrasèmes défigés des deux langues. 


\section{2 État de la question}

Le défigement est l'un des recours favoris aussi bien des journalistes, des publicistes que des écrivains, mais ce n'est que récemment que l'on y consacre des études spécialisées. On inscrit souvent ce recours dans un phénomène linguistique et stylistique plus large : celui des jeux de mots (e.g. Guiraud, 1979; Ben Amor, 2007).

Le terme de jeux de mots englobe toute sorte de transformations au niveau phonique, morphologique, lexical, phraséologique ou textuel. Ces transformations mènent inévitablement au déblocage de certains stéréotypes linguistiques et culturels. Pourtant, le jeu de mots s'étend aussi bien aux lexèmes qu'aux combinaisons de lexèmes (combinaisons libres ou contraintes), tandis que le défigement, en particulier, ne concerne que les modifications à l'intérieur des phrasèmes, ou énoncés non libres.

Il va de soi que certains outils conceptuels, élaborés pour traiter la question des jeux de mots, pourraient servir aussi dans l'analyse du défigement. L'opposition du ludant et ludé, proposée par Pierre Guiraud (1979), a été exploitée par beaucoup d'auteurs qui abordent le problème des jeux de mots ou du défigement (cf. de Foucault, 1988 ; Ben Amor, 2007 ; Blanco, 2013, etc). Inventés par analogie avec les termes saussuriens, le ludant qualifie le texte modifié qui subit le jeu, et le ludé, le texte d'origine. Le ludé est reconnu dans le ludant à l'aide du signal qui permet de rétablir la forme d'origine.

Le livre de Ben Amor (2007) présente une description minutieuse des jeux de mots dans les textes littéraires. L'auteure fonde son étude sur les romans de Raymond Queneau dont le style est ludique par excellence. Dans son travail, la chercheuse analyse à part le jeu de mots unilexical et le jeu de mots polylexical, ce dernier reposant souvent sur le défigement. L'auteure souligne l'importance primordiale du co-texte (contexte linguistique) et du contexte (composante extra-linguistique) dans la génèse et la perception des jeux de mots (Ben Amor, 2007 : 53, 64-65).

Le défigement, en tant que procédé autonome, a aussi été dégagé et étudié par les linguistes qui en proposent des approches et des classifications différentes. Parmi les typologies les plus élaborées concernant le défigement, mentionnons le classement des séquences défigées selon les types de modifications réalisées sur les séquences figées initiales. Ainsi, García-Page (1989) construit sa classification des expresiones fijas modificadas (expressions figées modifiées, EFM) dans la poésie autour des trois mécanismes formels de la mutation: altération de l'ordre des composantes des expressions figées, changement de l'inventaire des composantes (addition, suppression, séparation interne, commutation lexicale ou grammaticale) et opération transformative (nominalisation de l'unité phrastique, passivisation d'une structure active, etc). De même, Kouklina (2006), dans sa thèse, catégorise les expressions défigées dans la presse russe selon des ressources transformatrices de nature sémanticostylistique comme, par exemple, explication de la forme interne de l'expression figée, double actualisation, contamination phraséologique, ellipse phraséologique et d'autres.

La typologie de Rastier (1997) se fonde sur deux genres de critères: une typologie des moyens du défigement et une typologie des parcours interprétatifs. L'auteur attire l'attention sur la duplicité des parcours interprétatifs (une duplicité paradoxale, ludique ou satirique) quand deux interprétations, synthétique et analytique ${ }^{2}$, sont activées. Choisissant comme critère dominant le critère morphosyntaxique, Rastier indique des moyens de défigement tels que la modification syntagmatique de la lexie (inversion, intercalation), le défigement par contexte concurrent (reprise avec changement d'ordre, interaction entre lexies complexes, etc.) et d'autres.

Mejri (2009) dresse sa typologie selon les traits définitoires du figement, comme la fixité formelle et la globalité sémantique. Par conséquence, «toute atteinte de la fixité formelle et à la globalité sémantique des SF [séquences figées] serait considérée comme un défigement, ce qui produit des séquences défigées » (Mejri, 2009). Les types de défigement, relevant de la fixité formelle, se divisent selon le caractère de la fixité : fixité de nature phonétique, fixité morphologique, paradigmatique, syntagmatique, syntaxique, fixité d'actualisation, fixité de la combinatoire phrastique et de la combinatoire discursive. Le défigement relevant de la fixité sémantique implique la dualité littéral/global, la remotivation du sens littéral par association et remotivation par mention métalinguistique. 
Généralement observée depuis une perspective strictement linguistique (phraséologique et parfois stylistique), la facette littéraire du défigement, son aspect culturel et didactique ne sont pas non plus ignorés. La chercheuse polonaise Pajdzińska (1993) soumet à l'étude détaillée trois types différents de transformations des séquences figées dans la poésie polonaise contemporaine: dérivation à base phraséologique (derywacja odfrazeologiczna), contaminations des phraséologismes (kontaminacje frazeologizmów) et allusions phraséologiques (aluzje frazeoleogiczne). Sa méthode scientifique unit l'analyse linguistique à des déductions purement littéraires.

Du point de vue de Galisson (1994), c'est bien la référence culturelle qui est la clé de la compréhension des palimpsestes verbaux. Sa typologie de défigement s'organise, d'une part, autour de quatre axes principaux : délexicalisation avec ou sans filiation phonique et délexicalisation avec ou sans destruction syntaxique. D'autre part, Galisson propose une typologie des cultures mobilisées dans les palimpsestes, dont la culture cultivée (ou institutionnelle), culture culturelle (ou expérientielle), culture croisée (ou métissée). D'autres auteurs, comme, par exemple, Català (2012), soulignent aussi la portée didactique de l'étude du défigement.

Dans les articles récents de Blanco (e.g. 2012 et surtout 2013), consacrés au défigement dans la poésie française et hispanophone, l'auteur a pour point de départ les notions élaborées au sein de la TST (telles que phrasème, fonction lexicale, etc; cf. Mel'čuk, 2003) et la classification des locutions nominales établie par G. Gross (1996). La méthode d'analyse basée sur ces théories lexicalistes vise une description formelle et une cataloguisation précise des phrasèmes défigés et présente le défigement comme une manipulation linguistique avec un grand potentiel créateur dans un langage stylistiquement marqué comme celui de la poésie. C'est cette approche descriptive et appliquée à des textes poétiques que nous avons prise comme modèle pour notre recherche.

\section{Cadre théorique et notions de base}

Afin de délimiter notre champ de travail, c'est-à-dire, afin de le concrétiser, nous devons donner une définition du défigement. Cette notion, à son tour, ne serait pas correctement définie sans la délimitation du concept d'énoncé figé (ou contraint).

Dans notre étude, nous suivons, pour l'essentiel, la description du figement proposée au sein de la TST. Nous empruntons le concept de phrasème et surtout la classification des phrasèmes, exposée par Mel'čuk dans un de ses articles les plus récents (cf. Mel'čuk, 2013).

La notion fondamentale de la théorie mel'čukienne de figement est le phrasème. Les phrasèmes, selon Mel'čuk, sont des énoncés multilexémiques non libres. Un énoncé est considéré libre « si et seulement si il n'est pas contraint sur l'axe paradigmatique, c'est-à-dire si son sens et chacune de ses composantes lexicales sont sélectionnés par le Locuteur strictement pour ses propriétés linguistiques, c'est-à-dire indépendamment des autres composantes »(Mel'čuk, 2013 : 130). Dans le cas contraire, nous avons affaire à des énoncés non libres, i.e. à des phrasèmes.

Dans la classification de Mel'čuk, une collocation est un phrasème lexical ${ }^{3}$ compositionnel. Plus précisément, il s'agit d'un phrasème semi-contraint : une de ses composantes, la base de la collocation, est sélectionnée par le locuteur librement, pour son sens, tandis que le collocatif doit être choisi en fonction du sens à exprimer mais aussi en fonction de la base. Pour une description formelle des collocations, on applique l'appareil des fonctions lexicales $(=\mathrm{FL})$, un outil qui révèle la relation sémantico-lexicale entre la base de la collocation et son collocatif. Les collocations standard sont décrites par les FL standard, qui recouvrent un grand nombre de bases et produisent beaucoup de collocatifs : $\operatorname{Magn}($ silence $)=$ profond $; \operatorname{Sing}($ ail $)=$ gousse $\left[\right.$ de $\sim ; \mathbf{F u n c}_{0}($ soleil $)=$ brille $; \mathbf{O p e r}_{1}($ espoir $)=$ caresser $[\mathrm{ART} \sim]^{4}$. Quant aux collocations non standard, elles manifestent des liens sémantiques non systématiques entre la base et le collocatif, comme dans VIN blanc.

Les locutions, contrairement aux collocations, sont des phrasèmes non compositionnels. On les subdivise en trois sous-classes en fonction de l'inclusion du sens des composantes dans le sens de la locution (Mel'čuk, 2013 : 134). Ainsi, une locution forte n'inclut dans son signifié total aucun des sens de ses 
composantes : à titre d'exemple, le sens de la locution forte ${ }^{\ulcorner}$CINQUIEME ROUE DU CARROSSE ${ }^{7}$, 'une personne qui ne sert pas à grand-chose', n'inclut ni le sens de 'cinquième', ni de 'roue' ni de 'carrosse'. Une semi-locution inclut dans son sens le sens d'une de ses composantes, mais pas en tant que pivot sémantique, et n'inclut pas le sens de l'autre. En plus, un sens additionnel qui constitue le pivot sémantique de la semi-locution émerge. Le phrasème ${ }^{\Gamma F A I R E ~ L E S ~ Q U A T R E ~ V O L O N T E S ~}{ }^{7}$ [de N], au sens 'céder aux caprices de quelqu'un', correspond aux caractéristiques de la semi-locution. Enfin, une quasilocution renferme dans son sens le sens de toutes ses composantes, mais pas en tant que pivot sémantique, en incluant aussi un sens additionnel, qui joue le rôle de pivot sémantique, comme c'est le cas du phrasème 「PRENDRE LA VIE COMME ELLE VIENT?

Finalement, la classe des clichés regroupe, chez Mel'čuk, les pragmatèmes et les clichés proprement dits. Un cliché (e.g. Quelle heure est-il ?) est un phrasème sémantico-lexical compositionnel. La phrase Défense d'afficher (une inscription sur un mur signalant la prohibition) est un exemple de pragmatème, un type de cliché réservé à une situation concrètement définie.

Un proverbe représente un phrasème qui a une forme très contrainte, souvent archaïsée, comme dans les énoncés $A$ couur vaillant rien d'impossible. Il est à noter que les proverbes ayant un sens compositionnel entrent dans la classe des clichés, alors qu'il s'agit de locutions si leur sens est non compositionnel. Dans notre recherche, pour des raisons de commodité, nous rassemblons les clichés, les pragmatèmes et les proverbes (indépendamment du degré de compositionnalité) dans un bloc d'unités phrastiques, car ces trois types de phrasèmes constituent des énoncés autonomes.

Dans ce groupe d'unités phrastiques, nous incluons aussi des citations (ou formules identifiables) qui ne figurent pas dans la classification de Mel'čuk (2013) mais dont le défigement semble être un fait tout à fait habituel dans les textes littéraires. Par citations, nous comprenons toute sorte d'énoncés, plus ou moins clichéisés, dont l'origine et l'auteur sont identifiables : par exemple, la phrase On ne peut pas entrer une seconde fois dans le même fleuve, attribuée à Héraclite.

Les noms propres complexes, en tant que classe indépendante, sont aussi absents dans la typologie de la TST. Pourtant, constitués de deux éléments lexicaux ou plus, ces derniers imitent, en quelques sorte, la forme et les fonctions syntaxiques des locutions ou collocations. Le nom propre est défini, chez Jonasson (1994 : 21), comme «toute expression associée dans la mémoire à long terme à un particulier en vertu d'un lien dénominatif conventionnel stable». Nous considérons comme noms propres complexes les toponymes, les anthroponymes (réels et fictifs) et les titres ${ }^{5}$ multilexémiques des œuvres de littérature, peinture, cinéma, musique, etc. (e.g. Le déjeuner sur l'herbe, d'Édouard Manet).

À partir de toutes les notions introduites ci-dessus, nous définissons le défigement comme un recours linguistique qui consiste en la modification du signifié et, souvent, du signifiant d'un phrasème ayant pour résultat le déblocage de sa contrainte sémantique et syntaxique, déblocage qui provoque une collision des sens, synthétique et analytique. Signalons que le défigement est considéré comme tel à condition que l'énoncé final suggère au récepteur le phrasème initial.

\section{Typologies de défigement}

Il s'agit bien de typologies au pluriel, puisqu'il s'avère problématique de parler du défigement sans recourir à différents critères simultanément. À l'appui des différentes approches à l'étude du défigement et des diverses classifications proposées (García-Page, 1989; Kouklina, 2006; Ben Amor, 2007 ; Blanco, 2013), nous avançons trois typologies qui se complètent, ce qui nous aide à parvenir à une organisation et une description plus adéquate des exemples repérés dans les corpora.

Nous avons décidé, donc, de répartir les phrasèmes défigés, premièrement, selon le mécanisme de transformation qu'un phrasème initial subit (ou un phrasème source, si l'on emprunte le terme de Polguère, 2007); deuxièmement, selon la classe à laquelle un phrasème source appartient ; et, finalement, selon la catégorie grammaticale (G. Gross, 1996), ou la fonction syntaxique qu'un phrasème source a dans la phrase. Comme on peut le voir, tous ces classements partent du phrasème source et pas de l'énoncé cible (i.e. énoncé résultant). 
Bien que nous ayons choisi comme critère de classement la typologie des mécanismes du défigement, les deux autres typologies, selon la classe d'un phrasème source et selon sa fonction syntaxique, sont aussi explicitées (cf. les définitions entre accolades après chaque exemple). Notamment, pour le phrasème source, nous précisons sa classe et sous-classe sémantique, et nous distinguons entre les collocations (décrites par des FL standard ou non standard que l'on détermine pour chaque collocation), les locutions (locution forte, semi-locution, quasi-locution), les noms propres complexes (titre, anthroponyme, toponyme) et les unités phrastiques (proverbe, citation, pragmatème, cliché). En plus, pour les locutions et les FL, nous signalons leurs catégories grammaticales: nous parlons, donc, de locutions ou FL nominales, verbales, adjectivales ou adverbiales. Les unités phrastiques, ayant le comportement syntaxique d'une phrase, et les noms propres, qui sont nominaux par définition, sont exempts de cette dernière typologie.

Les mécanismes permettant le détournement des phrasèmes sont aussi appelés des procédés, ou des moyens de défigement. Ainsi, nous distinguons ${ }^{6}$ :

- La substitution lexicale, qui se produit quand une/des composante(s) d'un phrasème est/sont remplacée(s) par un/des élément(s) associé(s) à la/les composante(s) originaire(s) par des relations sémantiques (antonymie, synonymie, etc), des relations de paronymie ou homophonie, ou, éventuellement, des relations hors système :

Les ours se suivent et ne se ressemblent pas

(Les statuts de la liberté; Prévert, 1980)

$\{$ Phrasème source : unité phrastique type proverbe Les jours se suivent et ne se ressemblent pas; jours $\rightarrow$ ours : remplacement paronymique $\left.{ }^{7}\right\}$

Le plus souvent, mais pas toujours, cette substitution est «dictée» par le contexte ${ }^{8}$. Il arrive fréquemment que le phrasème source et l'énoncé résultant se rencontrent dans le même contexte et justement ce voisinage textuel produit un effet stylistiquement marqué, un effet d'analogie ou, au contraire, de contrepoids sémantique :

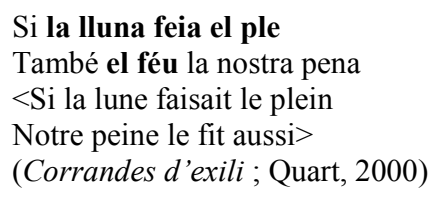

\{Phrasème source : collocation décrite par la configuration des FL standard $\left[\right.$ Culm + Func $\left.\mathbf{c}_{0}\right]($ lluna $)=$ fa el ple; lluna $\rightarrow$ pena : remplacement hors du paradigme sémantique dans le but de créer une analogie entre la phase culminante de la Lune et la culmination de la souffrance morale\}

- La libre saturation lexicale de la structure lexico-syntaxique rigide rappelle la substitution lexicale multiple et s'applique, normalement, aux unités phrastiques. Le moule syntaxique fixe et quelques éléments lexicaux, dispensés des manipulations, servent de signaux, c'est-à-dire, remplissent la fonction de clé du défigement :

\section{L'amour est mort vive la haine}

(La haine ; Brel, 1998)

\{Phrasème source : unité phrastique type pragmatème, propre à la sphère des cérémonies le Roi/la Reine est mort(e), vive le Roi/la Reine!; libre saturation de la structure lexico-syntaxique reconnaissable $N$. est mort, vive $N$.

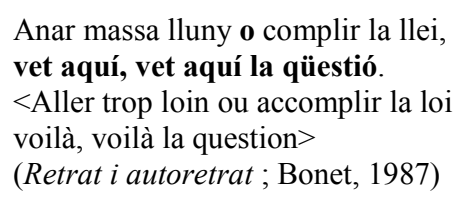


\{Phrasème source : unité phrastique type citation, extrait du $3^{\text {ème }}$ acte de la tragédie shakespearienne, To be, or not to be, that is the question; libre saturation de la structure $V_{I n f}+C o n j+V_{I n f}$ de la première ligne\}

- L'extension lexico-syntaxique causée par l'insertion dans un phrasème source d'un élément ou de plusieurs éléments (quelquefois, toute une proposition relative) altérant la combinatoire externe ou interne d'un phrasème source et détruisant ainsi son tout sémantique et syntaxique. Contextuellement conditionné, ce procédé « déclenche » un deuxième sens de l'une des composantes du phrasème ou de toute l'unité :

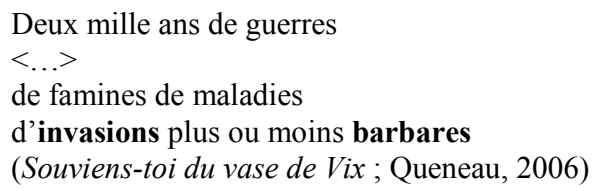

\{Phrasème source: quasi-locution nominale avec des propriétés dénominatives d'un nom propre ${ }^{\ulcorner}$INVASIONS BARBARES ${ }^{\urcorner} ; \Gamma^{\lceil I N V A S I O N S}+$ plus ou moins + BARBARES $^{\urcorner}$: extension interne $\}$

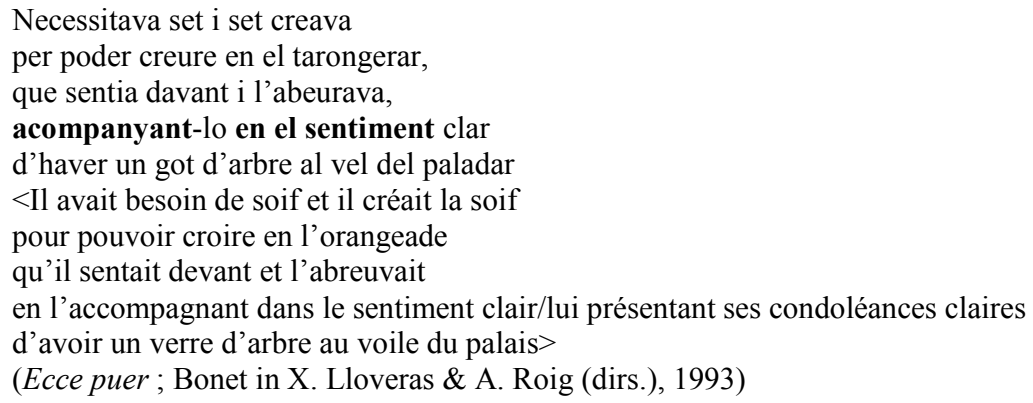

$\left\{\right.$ Phrasème source : quasi-locution verbale ${ }^{\ulcorner}$ACOMPANYAR $[\mathrm{N}]$ EN EL SENTIMENT ${ }^{\circ} ;\left\ulcorner^{A C O M P A N Y A R}[\mathrm{~N}]\right.$

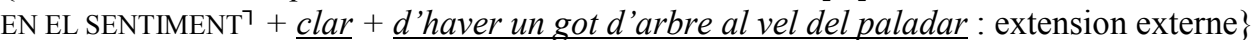

- L'intersection d'un phrasème avec un autre phrasème ou un syntagme libre; les énoncés qui se croisent partagent souvent une ou plusieurs de leurs composantes qui est/sont leur(s) axe(s) de croisement. Comme résultat de l'intersection, les contenus sémantiques des phrasèmes ou de quelques-unes de ces composantes se mêlent, et de nouveaux sens ${ }^{9}$ émergent :

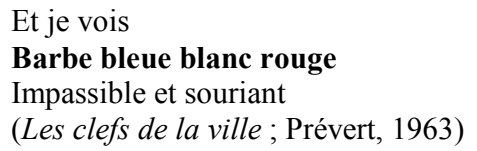

\{Phrasèmes sources: nom propre complexe, anthroponyme fictif Barbe Bleue + nom propre complexe, forme elliptique de la dénomination du drapeau républicain, Bleu Blanc Rouge ; axe de croisement : composante adjectivale bleu\}

Lors de l'intersection de deux séquences avec une composante verbale en tant qu'élément commun, on a souvent un zeugme pour effet stylistique, comme c'est le cas dans l'exemple catalan :

Escabellada, ronca,

perds la vergonya $i$ la senyera,

$<$ Décoiffée, enrouée,

tu perds la honte et le drapeau catalan>

(Oda a Barcelona ; Quart, 2000)

$\left\{\right.$ Syntagmes sources : collocation décrite par la FL verbale standard LiquFunc ${ }_{0}($ vergonya $)=$ perdre + syntagme libre perdre la senyera ${ }^{10}$; axe de croisement : composante verbale perdre\} 
- L'ambivalence forcée par le contexte qui se manifeste par l'emploi d'un phrasème dans un contexte qui force l'activation d'un deuxième sens (soit d'une de ces composantes, éventuellement des deux), tandis que le premier signifié reste aussi en jeu. Il s'agit, normalement, de l'activation des sens analytique et synthétique à la fois, sans, pour autant, rien changer dans l'inventaire ni dans l'ordre des composantes d'un phrasème :

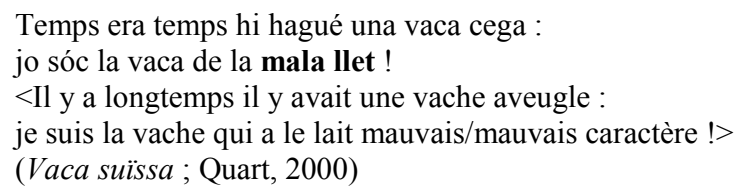

\{Phrasème source : semi-locution nominale ${ }^{\top}$ MALA LLET ${ }^{7}$; signifiés actualisés : 'mauvais caractère' et 'du mauvais lait'\}

- La commutation grammaticale a lieu quand le défigement est causé par le changement de catégorie grammaticale d'un phrasème ou d'une de ses composantes ou bien par le changement d'une signification grammaticale à l'intérieur de la même catégorie :

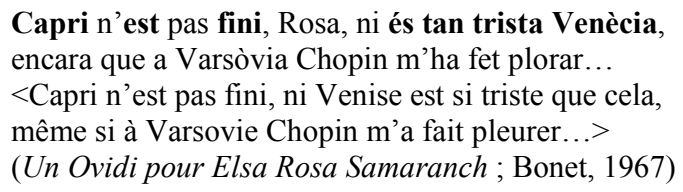

\{Phrasème source : nom propre complexe, titre de la chanson de H. Vilard Capri c'est fini; nom propre complexe, titre traduit de la chanson de Ch. Aznavour Que c'est triste Venise ; remplacement de l'affirmation par la négation\}

- Les modifications complexes qui regroupent plusieurs mécanismes à la fois. Des amalgames sophistiqués, formés à l'aide d'un ensemble de mécanismes, occasionnent l'actualisation de tout un nœud de sens, de connotations et de nuances sémantiques :

On fait force de trous dans ma lune de miel

(Le cocu ; Brassens, 1963)

\{Phrasèmes sources; locution forte verbale 「FAIRE UN TROU A LA LUNE`, quasi-locution nominale

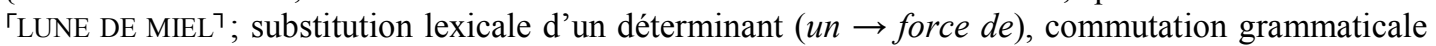
(s. trou $\rightarrow$ pl. trous), intersection de deux locutions avec l'élément lune comme axe de croisement\}

Dans ce travail, en addition aux typologies mentionnées, nous indiquons certaines caractéristiques des phrasèmes selon un critère stylistique qui pourrait, éventuellement, donner lieu à une typologie à part.

\section{Défigement comme procédé de (re)création linguistique dans la poésie : Exemples de phrasèmes défigés en français et en catalan}

Le défigement en poésie n'appartient pas au patrimoine d'un seul courant littéraire, d'un seul auteur ou d'une seule langue, et c'est ce que notre corpus de données multilingues confirme. Bien entendu, cela n'empêche pas qu'un auteur privilégie le défigement plus qu'un autre ou que des textes poétiques dans une langue comptent plus d'occurrences de ce phénomène que dans une autre. Ainsi, nous observons que, dans notre corpus poétique multilingue comptant plus de 1100 unités, les exemples de défigement en français sont les plus nombreux (400 unités environ), les données catalanes, au contraire, présentent un nombre plus réduit de cas de défigement (110 unités environ).

À notre avis, cette divergence quantitative n'est pas fortuite, et peut refléter la disproportion sociolinguistique qui existe entre le français et le catalan. Sans entrer dans les subtilités sociolinguistiques, nous supposons que l'insécurité linguistique des locuteurs d'une langue, comme le catalan (dont l'usage normal a été perturbé, au cours de son évolution, par certains facteurs historiques et 
politiques), pourrait expliquer l'aspiration prédominante (quoique pas tout à fait générale) à une manière poétique plutôt sérieuse que ludique.

Cependant, quelle que soit la dissemblance quantitative, les exemples en français et en catalan coïncident selon certains indices qualitatifs. Plus exactement, les mécanismes de transformations sont communs pour les deux langues et mènent à des métamorphoses sémantiques assez semblables. Par la suite, nous commentons quelques hypothèses de réorganisation sémantique des phrasèmes défigés.

\subsection{Double actualisation}

La double actualisation désigne, généralement, une sorte de polyphonie de sens, c'est-à-dire, un événement sémantique qui se produit lors de l'activation du sens aussi bien synthétique qu'analytique d'un phrasème. Plusieurs mécanismes de défigement aboutissent à cette double actualisation, mais, dans l'état le plus pur, on peut l'observer à travers un mécanisme que nous avons appelé ambivalence forcée par le contexte, un mécanisme qui ne prévoit aucune transformation syntaxique ou lexicale visible et où le contexte est le seul à offrir la clé du défigement :

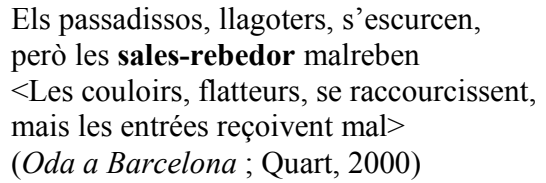

\{Phrasème source: collocation décrite par la FL adjectivale non standard SALA-rebedor; signifiés actualisés de la composante rebedor : 'qui sert d'antichambre' et 'qui reçoit'\}

Comme cet exemple le démontre, l'élément malreben agit comme un "déclencheur» d'un sens analytique dans la composante, employée, à l'intérieur du phrasème, au sens synthétique. Dans l'extrait ci-dessous, l'ambiguïté marque toute l'unité :

- Quel temps fait-il ?

- Il fait vite, mais si le cœur vous en dit il peut revenir sur ses pas.

(Un homme vient d'entrer... ; Prévert, 1980)

\{Phrasème source : unité phrastique type cliché Quel temps fait-il ? ; signifiés actualisés : 'je veux savoir quelles sont les conditions météorologiques' et 'je veux savoir à quel rythme il avance'\}

Il est clair que, dans la première réplique, le cliché porte le message conceptuel qui lui est propre, notamment, l'interrogation sur l'état météorologique. Pourtant, dans la réponse, le lexème TEMPS est utilisé dans le sens 'rythme, tempo', tandis que l'impersonnel il fait devient un prédicat avec son actant. On y voit donc l'altération du contenu conceptuel, sous l'impact du contexte, ce qui provoque le défigement.

La double actualisation correspond, dans les cas commentés, à la figure de syllepse de sens, qualifiée comme un procédé stylistique par lequel un mot est employé au sens propre et figuré à la fois (Dupriez, 1984 : 434). Guiraud (1979 : 11) définit ce type de jeu comme « une des formes cocasses du calembour polysémique » qui « consiste à bloquer le sens figuré en prenant l'expression au pied de la lettre ».

Signalons que presque tous les procédés de défigement impliquent, comme résultat, l'activation d'un deuxième sens au moins d'une des composantes du phrasème. La double actualisation, donc, peut être entraînée par d'autres mécanismes transformatoires, par exemple, par la substitution lexicale qui est un des mécanismes de défigement les plus productifs chez les poètes. Comparons, par exemple, ces deux extraits poétiques :

\footnotetext{
J'ai chanté la joie de vivre

En attendant celle de mourir

(Le troubadour; Brel, 1998)
} 
\{Phrasème source: quasi-locution nominale ${ }^{\Gamma \mathrm{JOIE}} \mathrm{DE} \mathrm{VIVRE}^{\urcorner}$; vivre $\rightarrow$ mourir : remplacement antonymique\}

\author{
Avui en terres de França \\ i demà més lluny potser, \\ no em moriré d'enyorança \\ ans d'enyorança viuré ${ }^{11}$ \\ $<$ Aujourd'hui sur les terres de France \\ et demain plus loin peut-être \\ je ne mourrai pas de nostalgie \\ je vivrai plutôt de nostalgie $>$ \\ (Corrandes d'exili; Quart, 2000)
}

\{Phrasème source : collocation décrite par la FL adverbiale standard Magn $($ enyorança $)=$ morir-se $[d$, $\sim$ ] morir-se $\rightarrow$ viure : remplacement antonymique\}

Dans les deux poèmes, les auteurs recourent à un cas spécial de substitution lexicale et font coïncider, dans la même phrase, les phrasèmes source et cible. La quasi-locution nominale 「JOIE DE VIVRE` est la source du défigement chez Brel. Grâce à la création de l'antonymie Anti(vivre) = mourir, les composantes du phrasème deviennent sémantiquement et syntactiquement indépendantes. Par conséquent, au lieu du signifié synthétique de la locution d'origine ('bonheur qui vient du simple fait d'exister'; cf. $\mathrm{TLF}_{\mathrm{i}}$ ), nous obtenons un signifié analytique, fabriqué comme résultat du défigement : 'joie d'être encore en vie'. Par contraste à cette signification analytique du phrasème source, la séquence cible joie de mourir désigne, certainement avec un ton ironique, le bonheur de ne plus vivre.

Dans la collocation catalane, dont le sens pourrait être transmis en français comme 'avoir une nostalgie implacable', s'effectue un changement d'accent sémantique: par l'impact du contexte et par une substitution antonymique réalisée dans la deuxième ligne (morir-se $\rightarrow$ viure), le collocatif morir-se prend sur soi le poids sémantique du phrasème, alors que normalement c'est la base ENYORANÇA qui devrait recevoir l'accent sémantique de toute l'unité collocationnelle. Vu ce transfert d'accent, la collocation morir-se d'ENYORANÇA acquiert une lecture analytique, 'perdre la vie à cause de la nostalgie'. Et justement, le poète affirme qu'il ne mourra pas de nostalgie, sinon qu'il vivra grâce à elle.

Il est à noter, qu'au niveau stylistique aussi, nous constatons un effet commun aux deux langues, celui qui correspond à la figure appelée chiasme, ou l'inversion de l'ordre des segments de deux groupes sémantiquement identiques (Dupriez, 1984 : 111).

\title{
5.2 Accommodation contextuelle
}

Dans les exemples précédents, c'est la composante verbale qui subit la substitution, en activant une signification inattendue, mais les corpora français et catalan fournissent aussi des exemples de transformation de quelques locutions verbales où c'est la composante nominale qui est remplacée par un terme choisi en fonction du contexte donné :

\footnotetext{
Au péril de mon cœur, la malheureuse écorne

Le pacte conjugal et me le déprécie,

Que je ne sache plus où donner de la corne

Semble bien être le cadet de ses soucis

(Le cocu; Brassens, 1963)
}

$\{$ Phrasème source : locution forte verbale $\ulcorner$ NE PLUS SAVOIR OU DONNER DE LA TETE $\urcorner$; tête $\rightarrow$ corne : remplacement méronymique\}

Selon Ben Amor (2007 : 231), l'élément nominal de la séquence verbale est, dans ce cas, le support de la transformation ludique. L'élément nominal ne dispose pas d'un signifié autonome au sein de la locution qui véhicule un sens synthétique déterminé. Ainsi, la locution 「NE PLUS SAVOIR OU DONNER DE LA TETE`?, avec le signifié total 'être débordé, avoir trop d'occupations, trop de choses à faire' (cf. TLF $\mathrm{i}_{\mathrm{i}}$, s'applique à toutes les situations où l'on a besoin de transmettre ce sens. Imaginons qu'il s'agit d'un mari cocu qui se 
plaint, outre des occupations quotidiennes, d'un plus grand souci, celui de l'infidélité de sa femme et de l'insolence de ses amants.

Devant ce défi poétique, Brassens utilise le moule syntaxico-sémantique d'une locution française, dont le sens synthétique est facilement reconnaissable, et il l'accommode à un contexte très concret en remplaçant un seul élément lexical, plus approprié contextuellement. Il existe, entre les éléments substituant et substitué, une relation qui n'est pas purement contextuelle, mais systémique : le lexème CORNE est le méronyme de TETE.

Chez Brassens, ce genre d'accommodation au contexte est l'un des procédés favoris et vise, bien entendu, un effet satirique ou, au moins, ironique. Ce n'est pas toujours le but pour d'autres poètes. Dans l'exemple catalan, l'objectif du poète est plutôt l'expressivité et la précision d'une image :

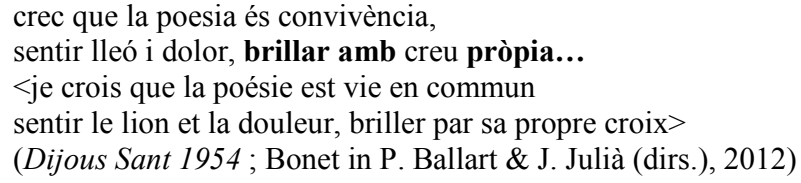

$\{$ Phrasème source : semi-locution verbale 「BRILLAR AMB LLUM PROPIA`; llum $\rightarrow$ creu $:$ remplacement hors du paradigme sémantique, dû à la tonalité religieuse de l'œuvre du poète\}

Le signifié de la semi-locution verbale ${ }^{\ulcorner B R I L L A R ~ A M B ~ L L U M ~ P R O P I A ~}{ }^{\urcorner}$, manipulée dans le premier extrait, est 'se distinguer par ses propres mérites'. Prenant en considération la thématique religieuse de la poésie de Blai Bonet, la creu qui substitue llum ne surprend pas, mais donne plus de vivacité à l'image. Faisons remarquer que l'accommodation au contexte, réalisée par l'auteur, aboutit ici à l'apparition d'une nouvelle connotation sémantique, fabriquée consciemment ou inconsciemment : la séquence défigée fait probablement référence aux épreuves pénibles réservées à un poète.

Le même déplacement de sens par ajustement au contexte n'affecte pas uniquement les locutions, mais aussi des unités phrastiques ou des noms propres complexes :

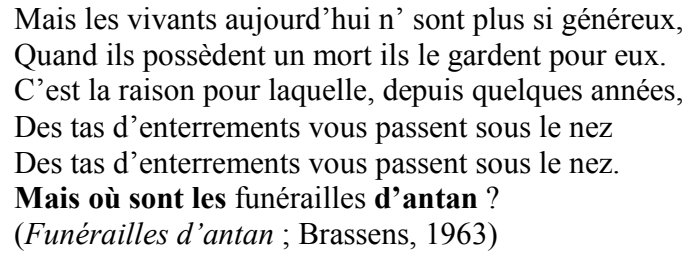

$\{$ Phrasème source : unité phrastique type citation, refrain du poème de Fr. Villon Ballades des Dames du temps jadis, Mais où sont les neiges d'antan ?; neiges $\rightarrow$ funérailles : remplacement contextuel\}

En catalan, nous retrouvons le même phénomène. Ainsi, Marta Pessarrodona i Artigues (Pessarrodona in P. Ballart \& J. Julià (dirs.), 2012), intitule le poème qu'elle a écrit en mémoire d'Amàlia Soler, Bella dama coneguda. La poétesse manie une dénomination typique des portraits de belles femmes dont le nom est resté inconnu pour l'histoire, Bella dama desconeguda $<$ Belle inconnue $\rangle$, en la défigeant par l'affirmation belle femme connue. Dans ce nom propre complexe, la composante adjectivale est substituée par son antonyme ${ }^{12}$ dans le but de nous faire comprendre que, contre toutes nos attentes, la dame, décrite dans le poème, n'est pas méconnue.

Comme le prouve ce dernier exemple, la composante nominale n'est pas le seul élément apte à être substitué afin de parvenir à un effet stylistique et sémantique. À titre d'exemple, chez Prévert, le déterminant, remplacé dans le collocatif, sert à adapter la collocation à la situation :

le bourreau et les jurés se ressemblent

comme treize gouttes d'eau

(Le pays des sosies; Prévert, 1980) 
$\{$ Phrasème source : collocation décrite par la FL adverbiale standard Magn (se ressembler $)=$ comme deux gouttes d'eau; deux $\rightarrow$ treize : remplacement contextuel du déterminant numéral\}

Ainsi, l'accommodation contextuelle est une sorte de conséquence sémantique du défigement que l'on atteint grâce à la construction d'un signifié sollicité par un contexte concret à partir d'un des sens d'un phrasème donné.

\subsection{Création d'une nouvelle valeur sémantique}

Les effets de défigement ne se réduisent pas à la concomitance des significations, enfermées dans les phrasèmes et leurs composantes, ni à l'ajustement de ces significations à un encadrement contextuel précis. Dans ces deux cas, on a affaire aux signifiés existants qui proviennent soit de l'ancienne métaphore et la forme étymologique effacée d'un phrasème, soit des sens désactivés de ses composantes, soit, dans les exemples les plus compliqués, d'une modification de sens imposée par le contexte avec la conservation d'un signifié initial. Tous ces effets sémantiques à part, le défigement peut engendrer des nœuds de sens très complexes, d'où émergent parfois des sémantismes imprévus, où l'on découvre non seulement des jeux entre les signifiés présents et camouflés, mais aussi la création de nouvelles connotations.

Les amalgames sémantiques naissent souvent de l'intersection de deux phrasèmes ou d'un phrasème avec un syntagme libre, ce qui mène à la soudure des signifiés des énoncés participants. Il est pertinent de citer à ce propos quelques lignes de Prévert :

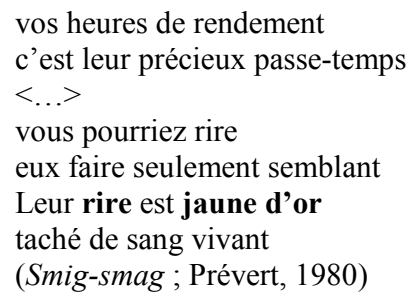

\{Phrasèmes sources: collocation décrite par la FL adjectivale standard AntiVer(rire) $=$ jaune + collocation décrite par la FL adjectivale non standard JAUNE d'or; axe de croisement: composante adjectivale jaune\}

Ici, les deux sens de l'adjectif JAUNE sont simultanément manipulés : le sens littéral, la première acception de l'adjectif ('qui constitue la couleur la plus chaude et la plus lumineuse et rappelle notamment la couleur du citron, de l'or, des blés murs', cf. $T L F_{\mathrm{i}}$ ), et un de ses sens figurés, qui, en combinaison avec le nom RIRE, le qualifie comme contraint, gêné. La collocation JAUNE d'or renvoie au sens propre de l'adjectif JAUNE, rapprochant la couleur jaune au ton de l'or. De toute évidence, Prévert fait référence à la couleur de la monnaie. Alors, si la collocation RIRE jaune désigne un rire perfide, forcé, la séquence soudée rire jaune d'or fabrique déjà un sens particulier à travers lequel on pourrait définir le rire perfide des riches. Cet énoncé défigé reflète l'attitude sarcastique et désapprouvante du poète envers les « économiquement forts».

L'exemple de Brassens montre comment le croisement du signifié d'un proverbe avec celui d'une collocation non standard attribue un nouveau sens au proverbe :

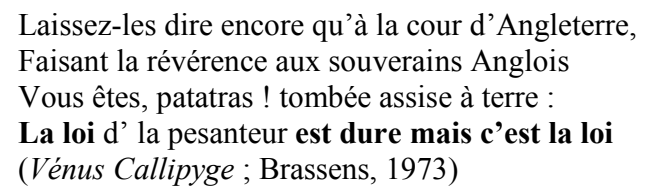

\{Phrasèmes sources : collocation décrite par la FL adjectivale non standard LOI de la pesanteur + unité phrastique type proverbe La loi est dure mais c'est la loi (lat. Dura lex sed lex); axe de croisement: composante nominale $l o i\}$ 
Le proverbe source rappelle qu'une loi, quoique dure, doit être respectée, tandis que la variante de Brassens spécifie, avec un grain d'humour, l'infaillibilité de la loi de la gravité. Le contexte prescrit la double entente de la composante dure: elle est comprise dans ce contexte comme 'pénible, rude à supporter', mais aussi comme 'dure, ferme', ce dernier sens étant dicté par le vers antérieur.

De la même façon, en catalan, les intersections de phrasèmes, renforcées, en plus, par d'autres modifications, causent des jonctions de sens :

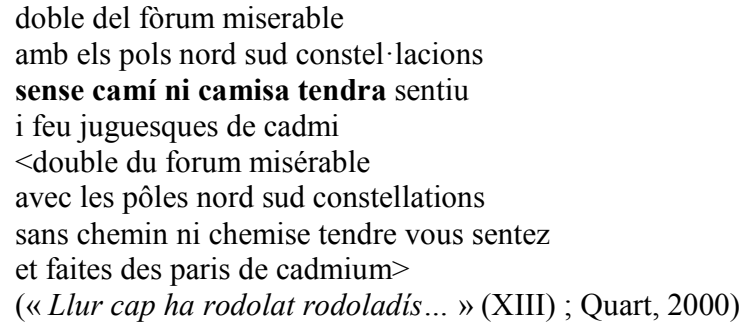

\{Phrasèmes sources: quasi-locution adjectivale 「SENSE CAMI + locution forte adjectivale 「SENSE CAMISA $^{\urcorner}$; intersection de deux locutions avec la composante prépositive sense comme axe de croisement; extension lexico-syntaxique externe : ‘SENSE CAMISA $\urcorner+$ tendra $\}$

Au niveau stylistique, l'intersection prend la forme de zeugme, une figure de syntaxe qui consiste à réunir plusieurs membres de la phrase au moyen d'un élément commun qu'on ne répétera pas ${ }^{13}$ (Dupriez, 1984 : 473). Au niveau sémantique, nous obtenons, comme dans les exemples français, un hybride : sense camí ni camisa tendra pourrait désigner 'égaré, privé de tout et misérable', un signifié qui collecte les sens synthétiques des deux locutions.

La fabrication d'un sens hybride ou d'une nouvelle valeur sémantique est quelquefois favorisée par d'autres mécanismes, comme la substitution lexicale :

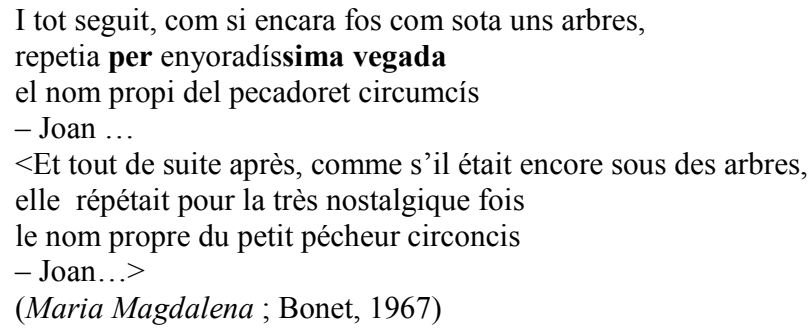

$\left\{\right.$ Phrasème source : quasi-locution adverbiale ${ }^{\lceil P E R}$ ENÈSIMA VEGADA ${ }^{\urcorner}$; enèsima $\rightarrow$ enyoradíssima : remplacement contextuel avec un changement morphologique du terme remplacé (enyoradissa $\rightarrow$ enyoradíssima, en superlatif), qui met en jeu aussi la paronymie (-èsima $\rightarrow-i$ ssima) $\}$

L'objectif sémantique chez Bonet semble plus transparent: si 「PER ENESIMA VEGADA` désigne plusieurs tentatives répétées d'affirmer ou de demander quelque chose, la variante détournée intègre dans ce signifié une nuance sentimentale, qui nous invite à comprendre repetir per enyoradíssima vegada comme 'répéter plusieurs fois et avec un peu de tristesse'.

Et mentionnons, pour conclure, une transformation sémantique résultant de la commutation grammaticale :

$$
\begin{aligned}
& \text { Je ne fais pourtant de tort à personne } \\
& \text { En suivant les ch'mins qui n' mèn'nt pas à Rome. } \\
& \text { (La mauvaise réputation; Brassens, 1973) }
\end{aligned}
$$

\{Phrasème source : unité phrastique type proverbe Tous les chemins mènent à Rome; remplacement de l'affirmation par la négation ; nominalisation de la structure phrastique\} 
Dans la version française du proverbe latin Tous les chemins mènent à Rome, nous rencontrons l'affirmation remplacée par la négation et, en plus, une transformation au niveau de la structure syntaxique : l'unité phrastique s'incorpore dans le texte en tant que groupe nominal, i.e. en tant que substantif avec une proposition relative. En fait, ici l'on peut remarquer aussi l'intersection du proverbe avec la collocation Real $_{1}($ chemin $)=$ suivre $[$ ART $\sim$ ] qui participe au défigement du proverbe mais ne le subit pas. Par les chemins qui ne mènent pas à Rome, Brassens comprend, vu le contexte de la chanson, son propre mode de vie différent de celui que la morale catholique et les mœurs de l'époque approuvent.

Des hybrides sémantiques et des tonalités supplémentaires, sans doute occasionnelles, qui apparaissent comme résultat du défigement, sont dotés d'une capacité mnémonique et, dans des conditions favorables, pourraient s'ancrer dans l'usage.

\section{Conclusions}

L'étude des textes en deux langues nous permet de conclure que le défigement s'effectue par des mécanismes communs et produit, dans les textes en français et catalan, des effets stylistiques semblables et parfois identiques. Cependant, si qualitativement et typologiquement les corpora dans les deux langues convergent, il existe des divergences quantitatives: les textes poétiques catalans offrent moins d'exemples de défigement que les textes français. Cela nous a menées à la conclusion que les poètes catalans analysés doivent préférer au défigement d'autres types de manipulations textuelles. Nous avons observé également dans nos corpora que les types de phrasèmes le plus souvent défigés sont les collocations et les locutions, alors que les unités phrastiques défigées sont un peu moins fréquentes et les noms propres complexes sont détournés plus rarement encore.

Au niveau du sens, l'effet de défigement peut être obtenu soit par la double actualisation d'une composante d'un phrasème ou de toute l'unité sous l'impact du facteur contextuel; soit par l'accommodation à un contexte concret moyennant une substitution à l'intérieur d'un phrasème source ; soit par la création d'une nouvelle valeur sémantique dérivée du croisement des signifiés initiaux entre eux ou de leur mélange avec des connotations contextuelles. En utilisant le défigement, comme procédé discursif, les poètes ont pour objectif aussi bien «l'affinage stylistique » (comme c'est souvent le cas des poètes catalans) que la réinterprétation humoristique des contraintes normatives du langage parlé ou littéraire (en quoi excellent les auteurs français) et, en tout, le « rafraîchissement » d'une image poétique à l'aide de manipulations autour du/des signifié(s) et signifiant(s) d'un phrasème.

Ainsi, le défigement décèle une importante dimension créatrice, hormis sa qualité de base qui est de « réveiller» des signifiés «dormants ». Il va sans dire, que le défigement peut offrir un instrument stylistique très puissant à un poète qui saurait le manier avec adresse.

\section{Remerciements}

Nous exprimons ici notre plus vive gratitude à Xavier Blanco Escoda pour ses commentaires précieux et la relecture minutieuse de la version finale de la présente communication.

\section{Références bibliographiques}

Ben Amor, Th. (2007). Le jeu de mots chez Raymond Queneau. Tunisie: Faculté des Lettres et des Sciences Humaines de Sousse.

Ben Amor, Th. (2006). Figement, défigement et jeux de mots formés sur énoncés proverbiaux. In François, J. \& Mejri, S. (dirs.), Composition syntaxique et figement lexicale, Caen : Presses universitaires de Caen, pp. 261-272.

Blanco, X. (2013). Le défigement des collocations comme recours stylistique. In Cahiers de Lexicologie. Revue internationale de lexicologie et de lexicographie, 102, pp. 207-225. 
Blanco, X. (2012). Le défigement des locutions nominales comme trait de style dans la poésie de Mario Benedetti. In Blanco, X. ; Fuentes, S. \& Mejri, S. (éds.), Les locutions nominales en langue générale, Bellaterra: Servei de Publicacions de la UAB, pp. 33-60.

Català, D. (2012). Figement et défigement des proverbes comme outil didactique du FLE. Paremia, 21, pp. 59-66.

Dupriez, B. (1984). Gradus : Les procédés littéraires (Dictionnaire). Paris : Union générale d’Éditions.

Galisson, R. (1994). Les palimpsestes verbaux : des révélateurs culturels remarquables, mais peu remarqués... Repères, 8, pp. 41-62

García-Page, M. (1989). Sobre los procesos de deslexicalización en las expresiones fijas. Español Actual, 52, pp. 5979.

Gross, G. (1996). Les expressions figées en français : Noms composés et autres locutions. Paris : Ophrys.

Guiraud, P. (1979). Les jeux de mots (Que sais-je?). Paris : PUF.

Jonasson, K. (1994). Le nom propre. Constructions et interprétations. Duculot : Louvain-La-Neuve.

Mel'čuk, I. (2013). Tout ce que nous voulions savoir sur les phrasèmes, mais... Cahiers de lexicologie. Revue internationale de lexicologie et de lexicographie, 102, pp. 129-149.

Mel'čuk, I. (2003). Collocations dans les dictionnaires. In Szende, Th. (réd.), Les écarts culturels dans les Dictionnaires bilingues, Paris : Honoré Champion, pp. 19-64.

Mel'čuk, I. (1997). Vers une linguistique Sens-Texte. Leçon inaugurale (faite le Vendredi 10 janvier 1997). Collège de France, Chaire internationale : http://olst.ling.umontreal.ca/pdf/melcukColldeFr.pdf

Mejri, S. (1997). Le figement lexical : descriptions linguistiques et structuration sémantique. Tunis : Publications de la Faculté des lettres de la Manouba.

Mejri, S. (2009). Figement, défigement et traduction. Problématique théorique. In Mejri, S. \& Mogorrón Huerta, P. (éds.), Figement, défigement et traduction $=$ Fijación, desautomatización y traducción, Alicante : Universidad de Alicante, pp. 153-163 : http://halshs.archives-ouvertes.fr/halshs-00617431

Pajdzińska, A. (1993). Frazeologizmy jako tworzywo współczesnej poezji. Lublin : AWH

Polguère, A. (2007). Soleil insoutenable et chaleur de plomb : le statut linguistique des greffes collocationnelles. In L'Homme, M.-C. \& Vandaele, S. (dirs.), Lexicographie et terminologie: compatibilité des modèles et des méthodes, Ottawa : Les Presses de l'Université d'Ottawa, pp. 247-291.

Rastier, Fr. (1997). Défigements sémantiques en contexte. In Martins-Baltar, M. (éd.), La locution, entre langues et usages, Paris : ENS Editions Fontenay / Saint Cloud, pp. 305-329.

Kouklina, I. (2006). Iavleniïa frazeologizatsyi i defrazeologizatsyi v iazyke sovremennoï pressy. Thèse de doctorat. Moskva : Moskovskiï Gosoudarstvennyï Oblastnoï Ouniversitet.

\section{Poésie citée}

Ballart, P. \& Jordi J. (dirs.) (2012). Paraula encesa : Antologia de poesia catalana dels últims cent anys. Barcelona : Viena Edicions.

Bonet, Bl. (1967). Evangeli segons un de tants. Barcelona : Proa.

Brassens, G. (1963). Georges Brassens par Alphonse Bonnafé. Paris : Éditions Seghers.

Brel, J. (1998). Tout Brel. Paris : Éditions Robert Laffont.

Desnos, R. (1953). Corps et Biens. Paris : Gallimard.

Lloveras, X. \& Roig, A. (dirs.) (1993). L'artista de la paraula : poesia catalana del segle XX. Barcelona : Proa.

Prévert, J. (1980). Soleil de nuit. Paris : Gallimard.

Prévert, J. (1963). Histoires. Paris : Galimard.

Quart, P. (2000). Poemes escollits. Barcelona : Edicions 62. 
Queneau, R. (2006). Courrir les rues. Battre la campagne. Fendre les flots. Paris : Gallimard.

\begin{abstract}
${ }^{1}$ Le choix des langues n'est pas arbitraire. Le français représente ce que l'on appelle la langue cultivée, i.e. une langue de grande diffusion et d'impact mondial important, alors que le catalan a le statut de langue minoritaire, une langue de diffusion restreinte qui s'emploie, en plus, dans des conditions de bilinguisme. Toutes ces caractéristiques peuvent influencer, par exemple, la fréquence d'usage du défigement dans des textes poétiques des langues respectives.
\end{abstract}

${ }^{2}$ À propos des interprétations analytique et synthétique, voir aussi Mejri (1997 : 593-597).

${ }^{3}$ Mel'čuk divise les phrasèmes, selon la nature des contraintes, en phrasèmes lexicaux et phrasèmes sémanticolexicaux. Les collocations et les locutions, les deux classes majeures de phrasèmes, appartiennent au groupe des phrasèmes lexicaux. Les phrasèmes sémantico-lexicaux forment la troisième classe majeure, celle des clichés.

${ }^{4}$ Pour une information plus détaillée sur les FL, consulter Mel’čuk (1996 et 2003).

${ }^{5}$ Il ne faut pas, pourtant, confondre les titres avec les citations : un titre est une dénomination, même s'il est multilexémique, tandis qu'une citation est, dans la plupart des cas de notre corpus, une unité phrastique qui a un comportement syntaxique similaire à celui d'un proverbe.

${ }^{6}$ Faute d'espace, nous ne mentionnons ici que les mécanismes les plus fréquents et les plus productifs.

${ }^{7}$ Cette ressemblance, en stylistique, s'appelle à-peu-près et désigne un double sens obtenu par un léger déplacement d'un ou deux phonèmes d'une phrase ou d'un syntagme et qui, d'après Dupriez, ne peut s'établir que dans le cadre d'une expression figée ou bien connue (Dupriez, 1984 : 59).

${ }^{8}$ Nous regroupons sous le terme contexte aussi bien l'encadrement linguistique (le co-texte) que la situation extralinguistique de l'énoncé défigé.

${ }^{9}$ L'intersection de deux syntagmes, dont au moins un est contraint, peut être accompagnée de perte d'éléments lexicaux qui ne participent pas dans la formation ou la réinvention des sens.

${ }^{10}$ Nous qualifions cet énoncé de non contraint, mais signalons que cette combinaison dénote une action symbolique : perdre le drapeau dans la bataille équivalait à perdre la bataille.

${ }^{11}$ Dans des cas pareils, l'inversion, l'anaphore et l'ellipse à l'intérieur de l'énoncé résultant sont justifiés et marqués stylistiquement grâce à la présence du phrasème source dans le contexte.

${ }^{12}$ Comme il s'agit, en fait, de la suppression du préfixe négatif des-, nous pouvons parler, dans ce cas, d'une commutation grammaticale.

13 Dans le $\mathrm{TLF}_{\mathrm{i}}$, la définition est plus précise du point de vue linguistique : le zeugma consiste à rattacher syntaxiquement à un mot polysémique deux compléments ou plus qui ne se construisent pas de la même façon ou qui ne correspondent pas au même emploi de ce mot. 